\title{
Extraspinal Percutaneous Osteoplasty for the Treatment of Painful Bony Metastasis
}

Jung Pil Yoon ${ }^{1}$, Kyung-Hoon Kiml, Young-Min Ok1 , Ji-Seok Baik², Tae-Kyun Kiml, Sang-Wook Shin', Hae-Kyu Kim²

${ }^{1}$ Department of Anesthesia and Pain Medicine, Pusan National University Yangsan Hospital, Yangsan, Korea

${ }^{2}$ Department of Anesthesia and Pain Medicine, Pusan National University Hospital, Busan, Korea

\section{Introduction}

- Extraspinal percutaneous osteoplasties (POPs) are novel techniques for the treatment of painful bony metastasis, which is often the cause of both persistent and incidental breakthrough pain.

- This retrospective study explored the efficacy, adverse effects, and complications of extraspinal POPs.

\section{Methods}

- Observational retrospective medical records were reviewed patients who received POPs from 2009 to 2015.

- Data collection: The origin of the cancer metastasis, performed POPs sites, coexisting sites of bony metastasis, the approach methods, necessity of adjacent joint injections, pain measurement, changes of VAS(visual analogue scale ) score and KPS (Karnofsky performance scale) score after 1 week of POPs, complications related to the POPs, and life expectancy

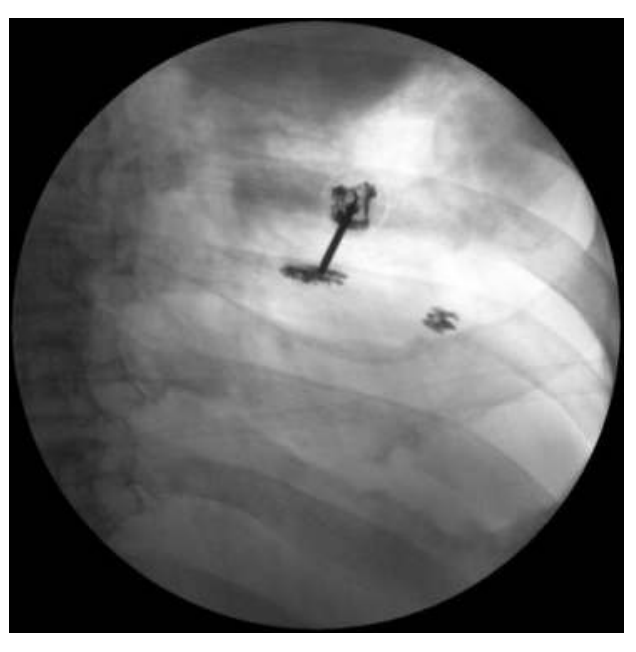

Fig.1. Costoplasty

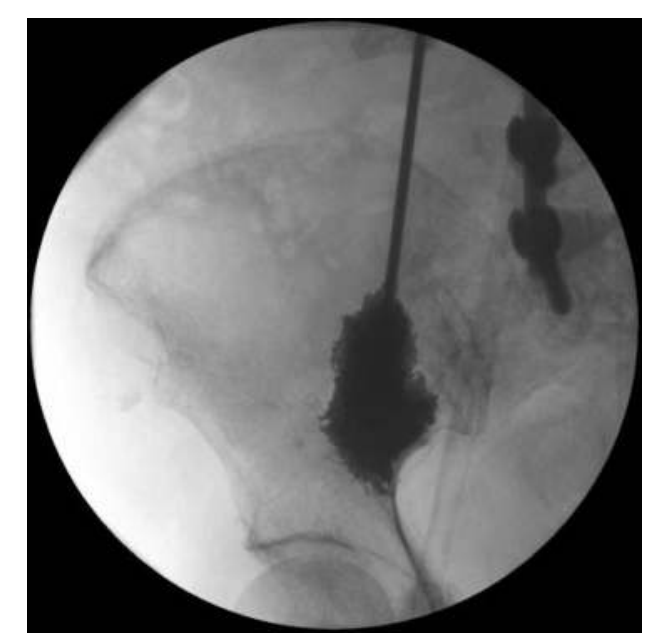

Fig. 3. Ilioplasty

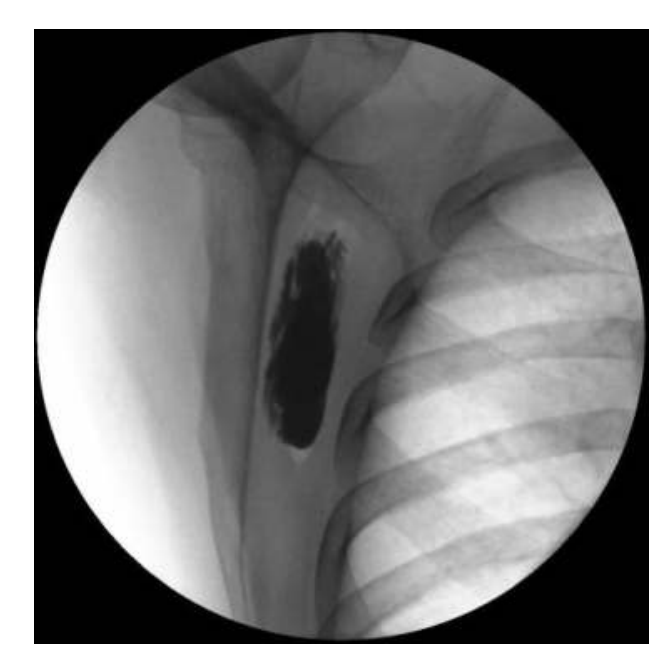

Fig. 2. Scapuloplasty

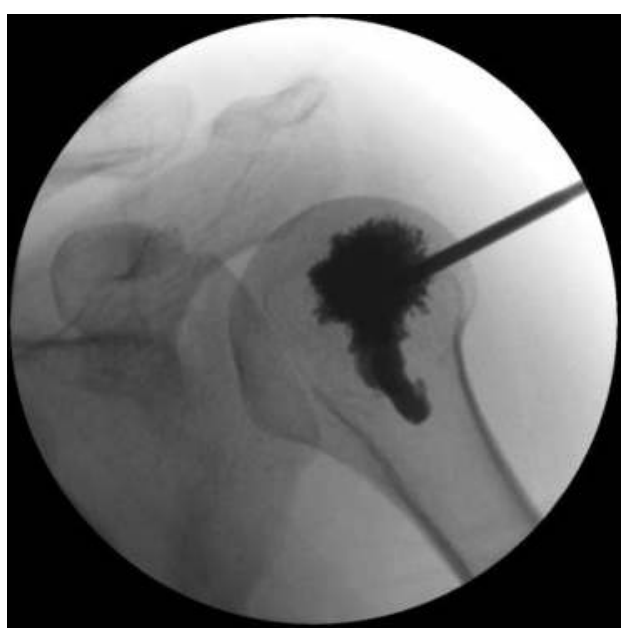

Fig. 4. Humeroplasty

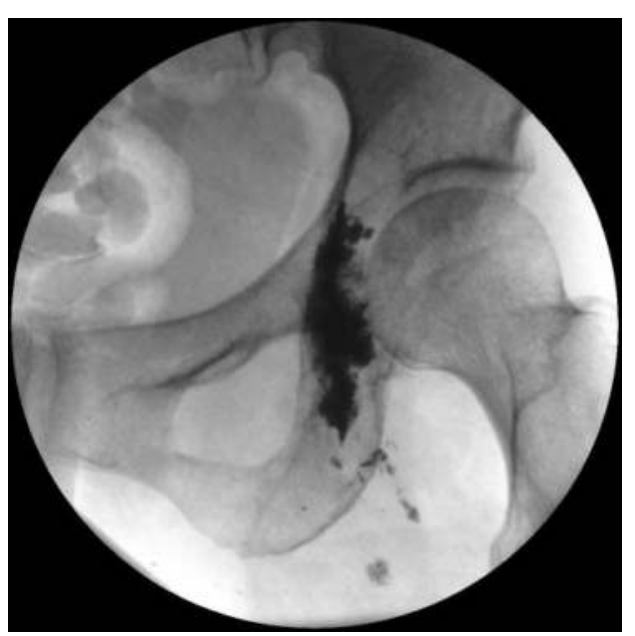

Fig. 5. Ischioplasty

\section{Results}

- $\quad$ A total of $48(\mathrm{M} / \mathrm{F}=27 / 21)$ patients had received 56 cases of POPs.

- Common sites for the origin of the cancer, in order of frequency: lung > liver> breast> colon>kidney.

- All patients who received POPs, excluding costoplasty and ischioplasty, needed adjacent joint injections before or after the POPs.

- Pain due to metastatic lesions was reduced significantly after the POPs immediately and sustained for the rest of their lives.

- The median KPS increased from $35.4 \%$ to $67.7 \%$ immediately after the POPs.

- There were no complications related to the procedures.

- The mean life expectancy after performing the POPs of patients who died afterwards (35/46), was 99.3 days, ranging from 1 to 767 days.

\section{Conclusions}

- Even though pain in the isolated POPs sites may be difficult to be measured with overlapping systemic pain, the POPs provided immediate pain relief and better physical performance when changing the position or loading their weight onto a dependent portion of body.

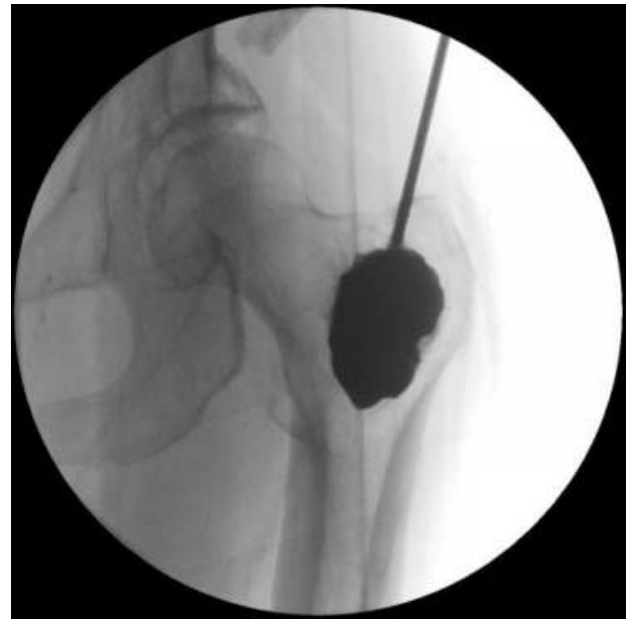

Fig. 6. Femoroplasty 